\title{
Beam Behaviour and Magnetic Field of K500 SCC
}

\author{
VINAY SINGH*, A DUTTA, A AGARWAL, C DAS, B NAIK, Z A NASER, S \\ PAUL, U BHUNIA, J PRADHAN AND J DEBNATH \\ Variable Energy Cyclotron Centre, 1/AF Bidhan Nagar, Kolkata 700064 \\ *Email:vsingh@vecc.gov.in
}

Received: May 7, 2015 | Revised: May 23, 2015 | Accepted: June 24, 2015

Published online: August 03, 2015

The Author(s) 2015. This article is published with open access at www.chitkara.edu.in/publications

\begin{abstract}
The extraction of the beam from the K500 Superconducting Cyclotron in Kolkata is posing a great challenge. After getting the internal beam, lots of effort were made to extract the beam and thereafter various experiments were designed and done to know the beam behaviour and to investigate the real reason. The deflector electrode was used in the Faraday cup mode. The deflector probe, bore scope probe and the main probe were used to investigate the beam behaviour by varying the first harmonic amplitude and phase with the help of trim-coil no. 13 by operating it in harmonic mode. Finally magnetic field mapping was done. In this paper, all these experimental results are described in detail.
\end{abstract}

Keywords: K500, Superconducting Cyclotron, Beam behaviour, Magnetic field mapping, Magnetic field imperfection

\section{INTRODUCTION}

K500 SCC got its first internal beam till the extraction radius in Aug 2009 [1]. This was confirmed by presence of neutrons in the neutron detector. The main probe and zinc sulphide screen (bore scope) were the only diagnostics available then. After installing the deflector, numerous runs were tried but the beam couldn't be guided through it. A series of experiments were designed to get into the bottom of the problem. It was then found that the magnetic field was one of the reasons behind this. This was further confirmed by magnetic field mapping. In this paper the steps leading to this conclusion are described in detail.

\section{THE ELECTROSTATIC DEFLECTOR}

\section{Deflector as a beam diagnostic element}

In K500 SCC there are two sets of deflector E1 and E2. The beam first encounters the deflector E1 which spans from $335^{\circ}$ to $34^{\circ}$. In the normal mode

\footnotetext{
Journal of Nuclear Physics, Material Sciences, Radiation and Applications Vol. 3, No. 1 August 2015 pp. 127-134
} 
Singh, V

Dutta, A

Agarwal, A

Das, C

Naik, B

Naser, ZA

Paul, S

Bhunia, U

Pradhan, J

Debnath, J

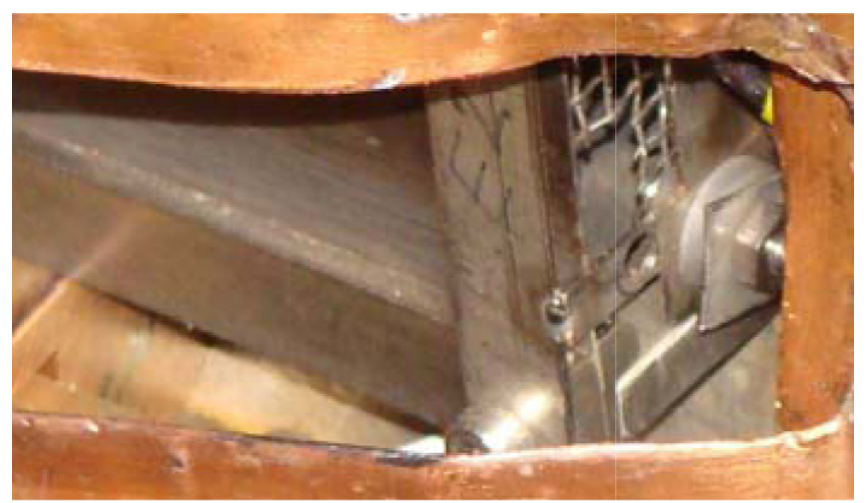

Figure 1: View of wire messed current diagnostic at the entry of Deflector.

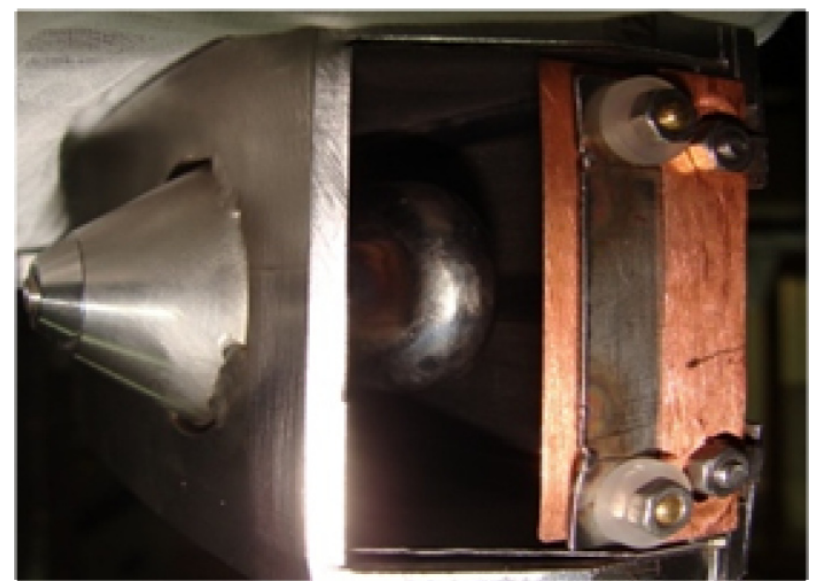

Figure 2: Solid copper block current diagnostic at the exit of Deflector.

i.e., when the power supply is connected to it, an electric field of around 2.5 $\mathrm{MV} / \mathrm{m}$ is created by applying high voltage of $20 \mathrm{kV}$ between the electrodes. The gap between them is $8 \mathrm{~mm}$.

To eliminate the possibility the beam was at all going through the deflector or not, the power supply was disconnected and the deflector was operated in Faraday mode i.e., as a beam diagnostic element.

The deflector E1 is not a fixed element, but has certain amount of freedom in respect to its radial distance from the centre of cyclotron. The entry position can be varied from 667 to $671 \mathrm{~mm}$ and the exit from 672 to $678 \mathrm{~mm}$. 


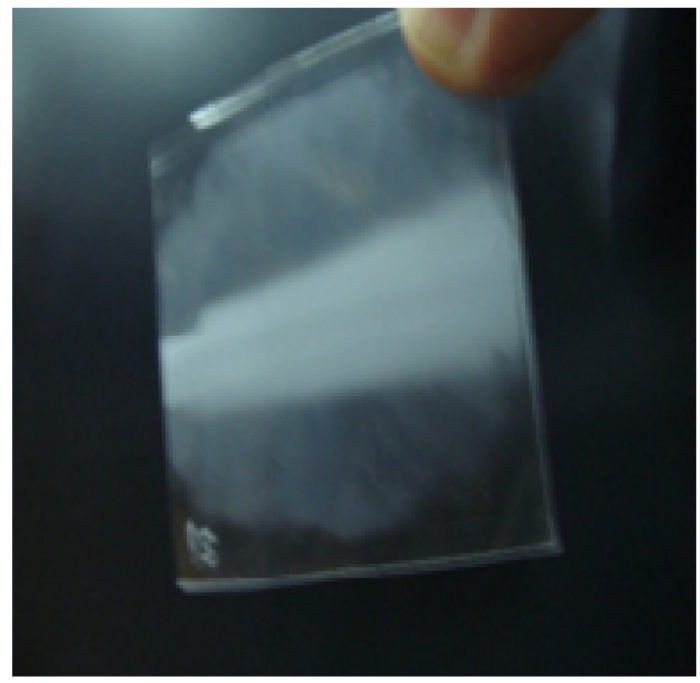

Beam Behaviour and Magnetic Field of K500 SCC

Figure 3: Beam mark on plastic sheet placed on deflector.

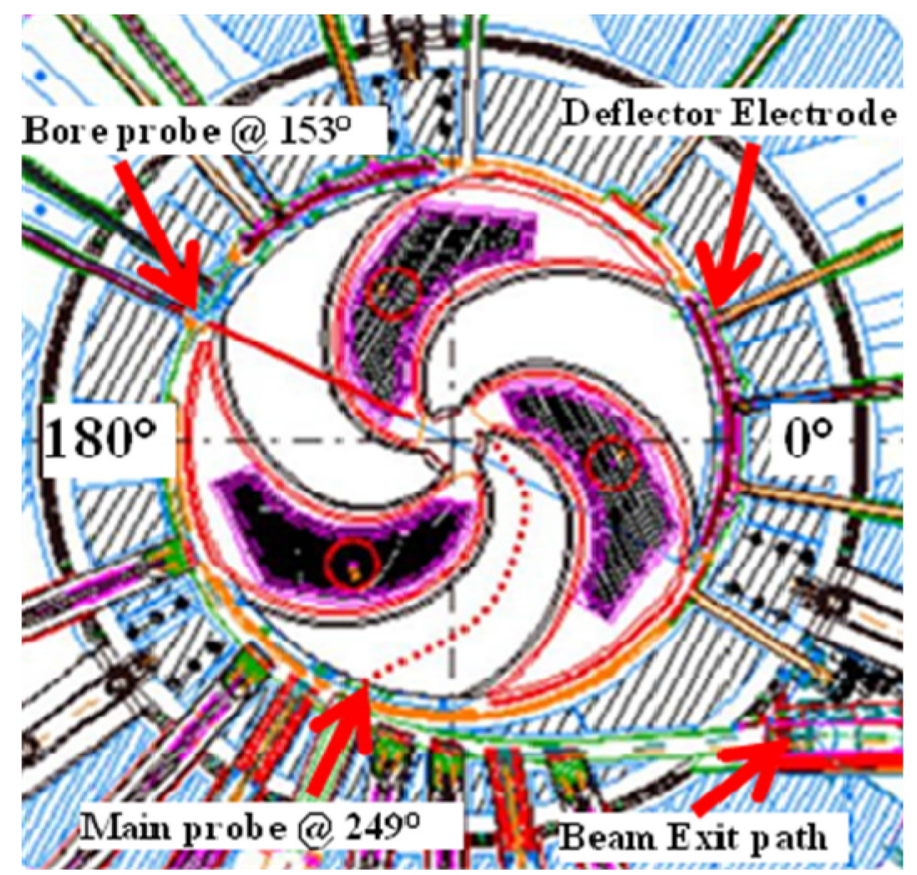

Figure 4: Median Plane top view with main probe, bore probe and deflector probe. 
Singh, $\mathrm{V}$

Dutta, A

Agarwal, A

Das, C

Naik, B

Naser, ZA

Paul, S

Bhunia, U

Pradhan, J

Debnath, J

The variation in position of deflector coupled with use of the $1^{\text {st }}$ harmonic coils (to slightly alter the beam curvature) was done repeatedly to get the beam signature on the outer electrode. However no significant beam current could be detected.

\section{Use of wire mesh at the entry and copper block at the exit of deflector}

To further investigate a wire mesh was placed at the entry of the deflector (Fig. 1) and a solid copper block at the exit (Fig. 2). This was done to ensure that any trace of beam reaching the deflector entry can be detected. And this beam if successfully passes through the deflector will be detected by the copper block. So, this time the deflector was used in the normal mode i.e., in the power supply mode.

Wide range of $1^{\text {st }}$ harmonic magnetic field amplitude and phase with the help of trim coil no. 13 was applied. This was done to vary the beam trajectory near the extraction zone so as to get beam signal from the entry mesh. However, no significant beam current could be detected. To further explore this point, the deflector voltage and its orientation were changed through a series of iterative methods, but no current could be detected on the exit copper block.

\section{Use of polymer sheets for Beam impression}

To get a further insight, another attempt was made by putting polymer sheets on the inner portion of the septum at three different locations - one near the entry, second at middle and the last near the exit. After a small amount of beam time the sheets were taken out for chemical analysis. The chemical analysis was done to exclude the possibility of any marks that could have arisen due to the high voltage discharge of the electrode. However, only a faint beam impression (Fig. 3) was found on the sheet placed near the deflector and no impression was found on the other two placed further away.

\section{BEAM CHARACTERISTICS USING PROBES}

The beam appeared to be not reaching the deflector side, but at the same time the beam was very much available towards the main probe and bore probe, so further investigations were continued to know the off-centeredness of the beam.

\section{Description of the Probes}

The K500 SCC has two kinds of probes- one is the main probe and other is the bore scope. The main probe is meant to read the intensity of current, whereas the bore scope is means to see the beam spot on a $\mathrm{ZnS}$ Screen. 


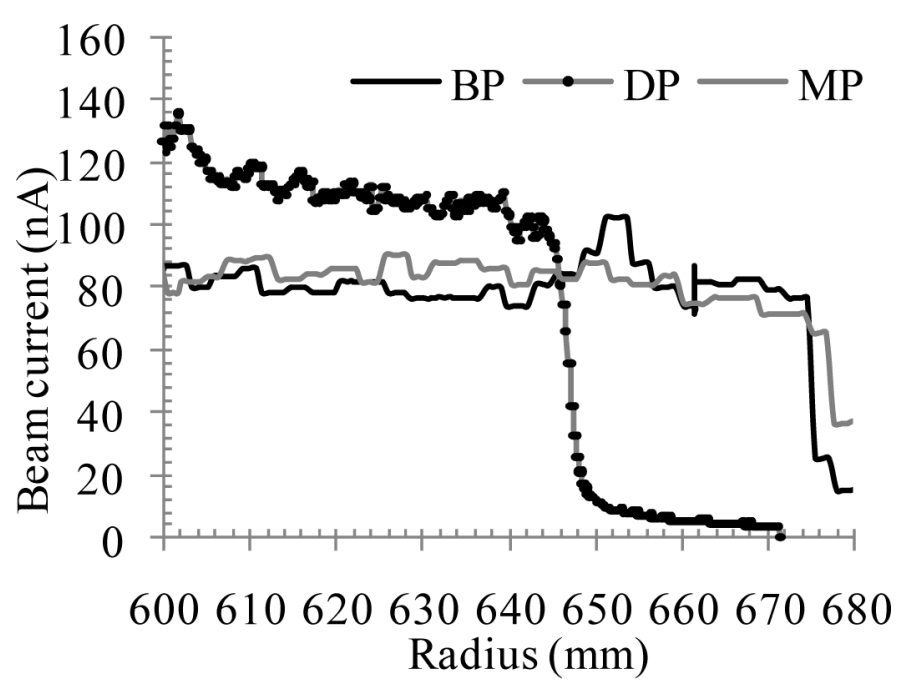

Beam Behaviour and Magnetic Field of K500 SCC

Figure 5: Beam profile on 3 probes without any external 1st harmonic.

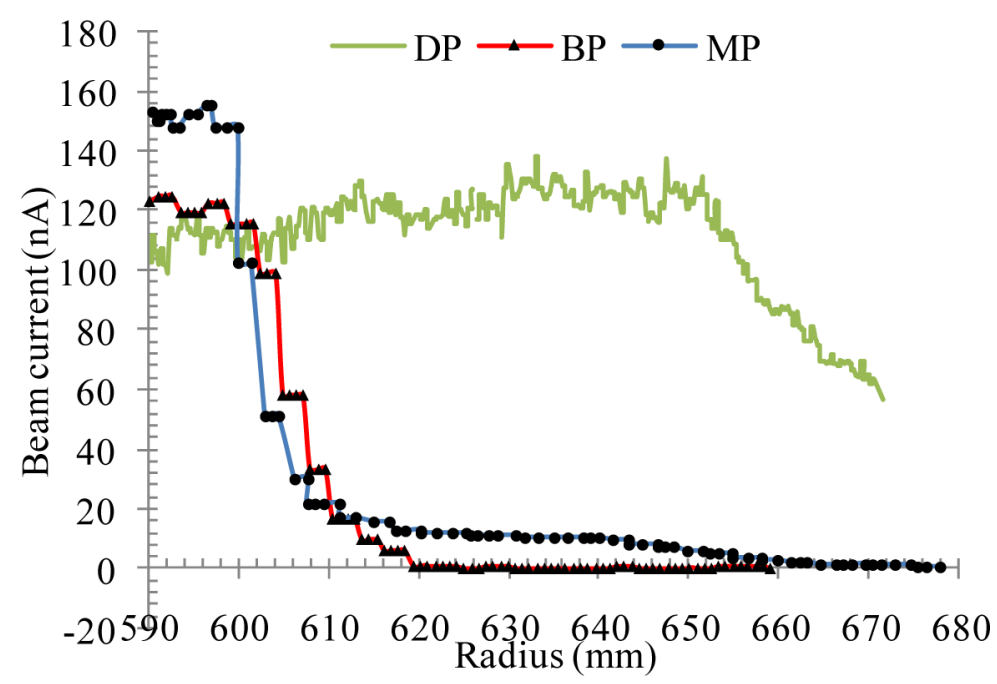

Figure 6: Beam profile on 3 probes with 15 Gauss 1st harmonic (phase $151^{\circ}$ ).

The main probe moves over the central spiral line of the hill whereas the bore probe moves across another hill after entering from its corner (Fig. 4).

To know whether the beam was shifting towards a particular area or not, need of a third probe was felt. For this reason the deflector was dismantled 
Singh, V

Dutta, A

Agarwal, A

Das, C

Naik, B

Naser, ZA

Paul, S

Bhunia, U

Pradhan, J

Debnath, J

(to gain access to a port) and another probe called the deflector probe was installed (Fig. 4) along $25^{\circ}$ azimuth.

\section{Scheme of measurement}

The beam curvature is not circular but a scalloped one. The local radius of curvature will thus change as the beam proceeds. The curvature will be less at the centre of the hill and more at the valley. Moreover, the three probes are not symmetrically placed with rest respect to the orbit trajectory. So, any data that is taken for the purpose of analysis has to take into account the scalloping of the beam vis-a-vis the position of the 3 probes.

\section{Profile of the Beam}

Beam profile with all the 3 probes was measured separately. It was observed that although good amount of beam was available up to the extraction radius $\sim 670 \mathrm{~mm}$ in main probe and bore probe, but in deflector probe the beam current was falling at $\sim 650 \mathrm{~mm}$ without application of any external $1^{\text {st }}$ harmonic field (Fig. 5).

This clearly indicated that beam was shifted towards main probe and bore probe region. Attempts were made to equalize the beam current in all the 3 probes by using different combinations of $1^{\text {st }}$ harmonic amplitude (up to 40 gauss) and phase $\left(1-360^{\circ}\right)$. One of those several iteration has been illustrated in figure 6 .

However, the distribution could not be equalized in all the three probes. From the shadowing of radial distribution of beam by using the said three probes, centering behaviour of the beam was quantified [2].

\section{MAGNETIC FIELD MAPPING}

These observations pointed out towards significant amount of imperfection in field and also towards the fact that this imperfection cannot be taken care of by the beam controlling parameters. Also through another experiment absolute phase measurement was done[3], which also pointed out towards the field imperfection. The remapping of the magnetic field was thus contemplated.

The magnetic field mapping was done by a search coil. The Calibration factor of the search coil was found out by the use of two NMR probes. The search coil was installed on a zig that was free to move in the horizontal plane. At any azimuth the search coil moved from -1.24 inch to 26.4 inches. The mapping was done for different excitations of main magnet coils namely alpha and beta coils 


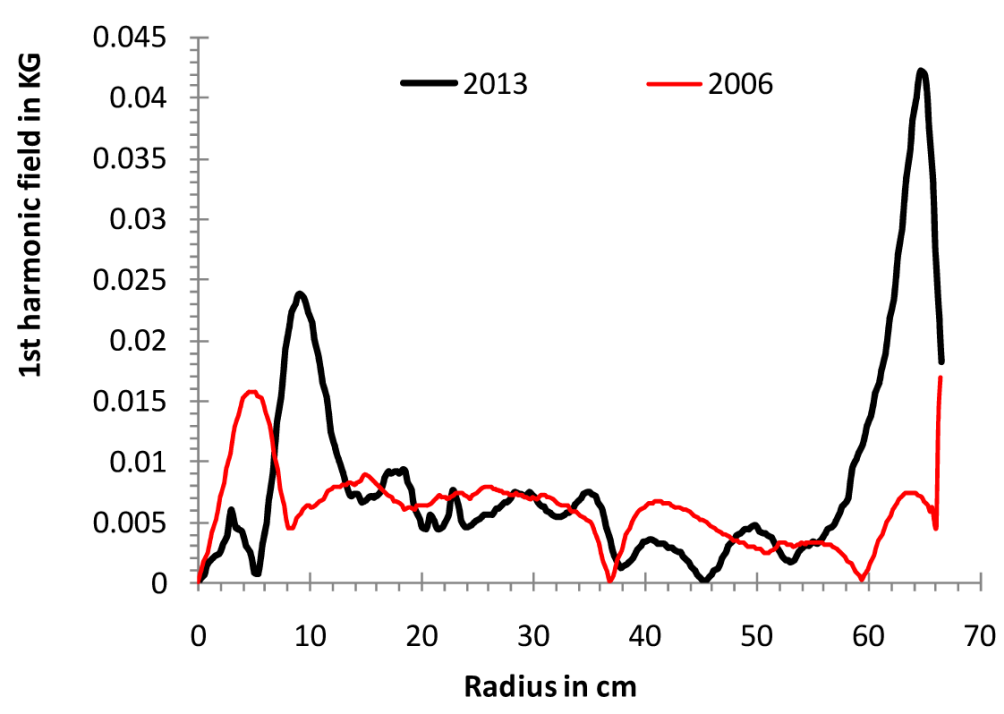

Beam Behaviour and Magnetic Field of K500 SCC

Figure 7: $1^{\text {st }}$ Harmonic field amplitude versus radius.

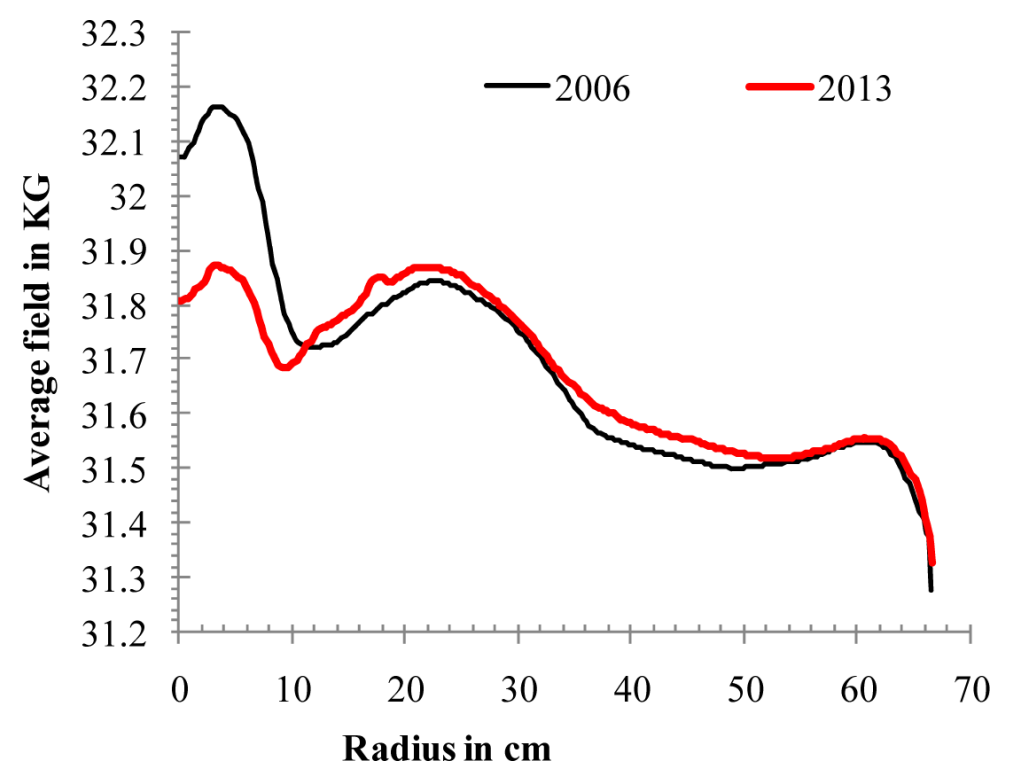

Figure 8: Average field versus radius.

The magnetic field mapping found that the average field near the central region was less by around 250 Gauss and the $1^{\text {st }}$ harmonic amplitude was quite high $\sim 45$ Gauss near the extraction region. 
Singh, V

Dutta, A

Agarwal, A

Das, C

Naik, B

Naser, ZA

Paul, S

Bhunia, U

Pradhan, J

Debnath, J

In figure 7 and 8 , the results of the magnetic field mapping is compared with that of the field mapping that was done earlier in year 2006.

\section{REMARKS}

The experimental observation that pointed out towards magnetic field imperfection was also thus verified by remapping of the magnetic field. The correction of magnetic field is currently being done.

\section{ACKNOWLEDGMENT}

The authors would like to thank all persons who have contributed directly or indirectly, especially the control room personnel who have toiled through the day and the night in the endeavour towards a common goal.

\section{REFERENCES}

[1] C. Mallik et al, "First Beam Acceleration in Kolkata Superconducting Cyclotrons," Proceedings of CYCLOTRONS 2010, Lanzhou,China.

[2] J Debnath et al, "Beam dynamics in presence of imperfection fields near the extraction zone of Kolkata superconducting cyclotron," Proceedings of CYCLOTRONS 2013, Canada.

[3] T Bhattacharjee et al, "Development of a fast scintillator based phase measurement system for compact superconducting cyclotrons," Rev. Sci. Instrum. Volume 84, Issue 5 (2013). http://dx.doi.org/10.1063/1.4807076 